\title{
Modeling of the light scattering properties of cometary dust using fractal aggregates
}

\author{
I. Bertini ${ }^{1}$, N. Thomas ${ }^{2}$, and C. Barbieri ${ }^{1}$ \\ 1 Dipartimento di Astronomia e CISAS, Università di Padova, Vicolo dell'Osservatorio 2, 35122 Padova, Italy \\ e-mail: bertini@iaa.es \\ 2 Physikalisches Institut, Space Research \& Planetary Sciences, Sidlerstrasse 5, 3012 Bern, Switzerland
}

Received 19 April 2006 / Accepted 30 August 2006

ABSTRACT

\begin{abstract}
Aims. The nature of cometary dust is still elusive. In order to constrain the physical properties of dust in cometary coma, we modeled the observed scattering parameters, investigating different morphological and compositional effects.

Methods. We used fractal aggregates as dust particles. The scattering behavior of extremely elongated dust particles, similar to those formed in micro-gravity aggregation experiments and more compact aggregates, have been studied with the discrete-dipole approximation theory.

Results. Results show that particles comparable in size to the incident wavelength provide the best qualitative fits. A silicate composition gives a good fit to the polarization and the phase function, but is characterized by large albedo. Organic and mixed compositions provide low albedos but do not fit the polarization and phase function curves well. Compact particles seem to reproduce the observed parameters better, indicating a possible morphological metamorphism of dust since the time of formation. The observed different polarization classes may be explained in terms of different monomer sizes, if a cluster of spheres models are used.
\end{abstract}

Key words. scattering - comets: general

\section{Introduction}

The scattering properties of cometary dust provide important information about the physical characteristics of the dust itself because the scattering of the incident unpolarized sunlight depends on the morphology and the composition of the dust particles. The observed scattering properties for different comets appear to be roughly similar and can be summarized as follows.

The linear polarization, $P$, of the scattered light is a bellshaped curve with a maximum at intermediate phase angles, $\alpha$, $\left(\alpha=90^{\circ}-100^{\circ}\right)$. At low-phase angles all the comets share similar polarization values, and $P$ shows a negative branch with an inversion angle near $21^{\circ}$ and a slope of $\sim 0.2 \%$ per degree at the inversion angle (Levasseur-Regourd \& Hadamcik 2001). The negative branch has an approximately symmetric shape and a minimum of $\sim-2 \%$ near $10^{\circ}$. For $\alpha>30^{\circ}-35^{\circ}$ the comets observed in the visible wavelength range can be divided into three classes: comets with low polarization maximum, $P_{\max }$, of $10 \%$ to $15 \%$ and comets with high polarization maximum of $25 \%$ to $30 \%$ and comet Hale-Bopp that shows the highest polarization observed by far in a comet (Levasseur-Regourd \& Hadamcik 2001). For $\alpha>30^{\circ}-40^{\circ}$, the polarization at a given phase angle increases with the wavelength. This is the so-called polarization color effect (Levasseur-Regourd \& Hadamcik 2001).

The origin of the three polarization classes is still puzzling. It has been shown by Chernova et al. (1993) that comets with high $P_{\max }$ are dust-rich, while comets with low $P_{\max }$ are gasrich and dust-poor. Moreover, Levasseur-Regourd et al. (1996) and Hanner \& Hayward (2003) have noticed that comets with high $P_{\max }$ exhibit silicate emission features, while these features are absent in other comets. The existence of the polarization classes may be related to differences in the bulk properties of the dust, such as albedo, size distribution, and porosity (Levasseur-Regourd et al. 1996). On the other hand, Kiselev et al. (2004) underline the problem of gas contamination in polarimetric measurements with wide-band filters and consequent depolarizing effects on light scattered by dust. It is therefore possible that the trichotomy in polarization is an artefact and that the cometary dust in the coma is characterized in all cases by high values of $P_{\max }$.

Kelley et al. (2004) have shown that the polarization of comets in the near IR (up to the $K$ band) shows a weak trend to higher values at higher wavelengths, resembling the color effect observed at visible wavelengths. Comets appear to divide into a high and low polarization class as well.

The behavior of the negative branch in the IR region is less clear. Only two comets have been studied polarimetrically in the IR at small phase angles: Halley and Hale-Bopp. Halley has been studied in the IHK bands by Brooke et al. (1987). Halley showed an increase in the polarization with $\lambda$ at large $\alpha$, while there was no such trend at small phase angles. The comet clearly exhibited a negative branch of few $\%$ in the three bands. On the other hand, measurements of the polarization of comet Hale-Bopp at $1.5,1.7$, and $2.2 \mu \mathrm{m}$ by Jones \& Gehrz (2000) showed no evidence of a negative branch. Because of these uncertainties, one of the purposes of our study was the investigation of the effect of different bulk properties of the dust (such as morphology and composition) and the size distribution on the polarization in order to understand better which parameters might reproduce the three observed polarization classes.

The scattering phase function, $\Phi_{S}$, possesses a distinct forward scattering surge, a rather gentle backscattering peak, and a flat shape at medium phase angles (Kolokolova et al. 2004). Observations of many comets give a phase function 
enhancement in the forward direction, hereafter FSE (forward scattering enhancement), about a factor of 10 as $\alpha$ increases from $80^{\circ}$ to $150^{\circ}$ (Ney \& Merrill 1976; Ney 1982; Gehrz \& Ney 1992). The increase in the backscattering direction, hereafter BSE (back scattering enhancement), can be measured as the ratio of the phase function at $\alpha=0^{\circ}$ and $30^{\circ}$. Some comets show $B S E \sim 2$ (Millis et al. 1982; Hanner et al. 1985). Only a few data are available at high-phase angles because of the angular proximity of comets to the Sun.

As summarized in Kolokolova et al. (2004), the coma dust particles are characterized by low geometric albedo, generally $<0.06$ (Kimura et al. 2003). The low values are derived from observations (e.g. Hanner et al. 1985; Tokunaga et al. 1986; Fulle et al. 2000).

The scientific goal pursued in this paper is to study the scattering behavior of dust particles with different morphologies and compositions to model the observed scattering properties and to compare the results with the present knowledge of cometary dust. Dust particles as fractal aggregates of spherical basic constituents, called monomers, have been studied. The dust particles have mainly a size comparable to visible wavelengths. Their scattering behavior was analyzed with the discrete-dipole approximation (DDA) theory. We investigated the properties of elongated dust particles, characterized by a low fractal mass dimension, similar to that produced in the pre-planetary dust aggregation experiment CODAG (Krause \& Blum 2004), hereafter particle-cluster aggregate (PCA) particles, and made a comparison of their scattering behavior with more compact ballistic particle-cluster aggregate (BPCA) particles. The investigation was performed at $0.535 \mu \mathrm{m}$ and $0.6274 \mu \mathrm{m}$, corresponding to the peak transmission wavelengths of the Rosetta spacecraft's OSIRIS Wide Angle Camera green and red broadband filters (Keller et al. 2006). These values are also close to the Johnson $V$ and $R$ filters normally used in astronomical observatories. Since polarimetric measurements of the dust coma at IR wavelengths, where the dust particles sizes may be more comparable to the incident wavelength, can further constrain the nature of the cometary grains (Kelley et al. 2004; Petrova et al. 2004), we also ran sample simulations in the IR band. The wavelength selected was $1.5 \mu \mathrm{m}$ because, in general, the laboratory data required to model the dust composition extend up to a wavelength of $1.5 \mu \mathrm{m}$. The scattering analysis was performed by studying the phase angle dependence of the polarization and phase function curves for single, independent dust particles with a specified shape and composition. Moreover, the geometric albedo was also calculated and the results averaged over a particle size distribution in order to obtain a more realistic picture of cometary dust. Our analysis was compared, where possible, with previous theoretical and experimental light scattering works, although different morphologies, compositions (mainly silicates and carbonaceous components), and scattering techniques were used.

\section{The morphology}

It has been shown that the observed scattering properties and the IR spectra of comets can be reproduced by clusters of spheres or irregular particles that can be described in fractal terms (for a short summary of the scattering study results see Mann et al. 2004, while for IR spectra fits see Hayward et al. 2000; Moreno et al. 2003). Moreover, interplanetary dust particles (IDPs) of cometary origin are characterized by a fluffy, highly porous, aggregate structure (Brownlee et al. 1994). The CODAG experiment on pre-planetary dust aggregation showed that elongated dust chains form in a micro-gravity regime. These structures are characterized by a low fractal mass dimension, $D_{\mathrm{f}} \sim 1.4$ (Krause $\&$ Blum 2004). Although a morphological and compositional metamorphism might take place inside comets, it is possible that cometary dust maintains its pristine nature because in the protosolar nebula the dust particles were completely preserved during the aggregation into comets (Greenberg \& Hage 1990). This would imply that dust aggregation experiments provide direct information about the particles released from the cometary nucleus.

In order to model and study dust particles with a low fractal mass dimension, a code to generate aggregates randomly using constituent spheres was developed. We have used particle-cluster aggregation under some assumptions. The generation mechanism is quite straightforward. The first monomer of the chain is placed at a fixed point in a 3D grid. The next monomers are then randomly generated on a sphere centered at the coordinates of the first monomer and are forced to move in the direction of the closest one, sticking with it at the contact point. In this way random chains with an elongated structure are obtained. The code to obtain more compact BPCA particles is a modification of an original code provided by T. Kozasa (Kozasa et al. 1992, 1993). The BPCAs have been produced by shooting projectiles (constituted by a single monomer) onto a target at random, one at a time (Kozasa et al. 1992). The trajectories of the projectiles are assumed to be ballistic. In the aggregation process, the projectiles are attached at the point where they hit the target. Examples of the generated PCA and BPCA dust particles with different number of monomers, $N$, are shown in Fig. 1.

After the generation of the dust particles, it is possible to derive their morphological characteristics, such as the fractal mass dimension, $D_{\mathrm{f}}$ (Wurm \& Blum 1998). Using the average value of $10^{5}$ dust particles with the same $N$ for $N=$ [5-100], corresponding to the size range used in the scattering simulations, we find $D_{\mathrm{f}}=1.26$ for PCA and $D_{\mathrm{f}}=2.71$ for BPCA particles. Since the PCA particles have low $D_{\mathrm{f}}$, comparable with the results of the CODAG experiments, they can be used to model the structure of loose pre-planetary dust particles.

The morphological structure of the dust particles is related to their porosity, $p$ (Kozasa et al. 1992). In Table 1 values of $p$ for $N=5,15,30,50,100$, and both classes are shown. As can be seen, PCA and BPCA dust particles are characterized by high porosity. The aggregates with lower $D_{\mathrm{f}}$ have higher porosity than more compact aggregates. The value of $p$ increases with $N$ for both classes.

\section{The composition}

Data from space missions, together with ground-based spectroscopic observations of comets, the analysis of IDPs with a supposed cometary origin and theoretical modeling of light scattering from cometary dust, constrain the component materials.

The mass spectrometer PUMA-1 onboard Vega-1 (Jessberger 1999) gave evidence that cometary dust grains are mixtures of two end-member components, called CHON and Rocks. Rock-rich, CHON-rich, and Mixed particles were detected. Rock and CHON do not occur as pure components but are intimately mixed down to the finest scale (Lawler \& Brownlee 1992). The CHON component is mainly organic refractory material (Jessberger et al. 1986; Kissel \& Krueger 1987). The Rock component is mainly composed of Mg-rich, Fe-poor silicates (Jessberger 1999). More recently, a preliminary analysis of the results of the NASA Stardust mission to comet Wild 2 showed that the dust composition of Wild 2 is similar to the one derived from the Halley flyby, independent 


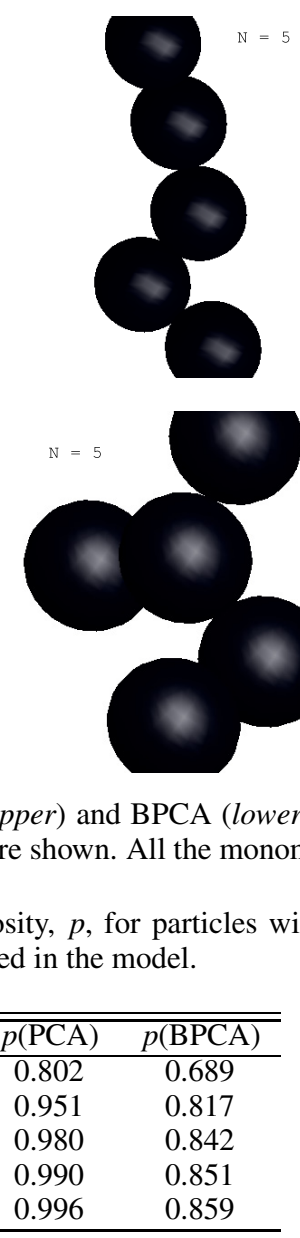

of the different age, the sampled position, and the level of cometary activity (Kissel et al. 2004). Mg-rich silicates have been also used to model silicate emission features in the IR ground-based spectra of comets (e.g. Colangeli et al. 1995, 1996; Wooden et al. 1999; Hayward et al. 2000; Harker et al. 2002). The modeling of observed IR spectra of comets with laboratory data suggests silicate and carbonaceous components (Harker et al. 2002). Moreover, the IDPs with a cometary origin confirm the presence of silicates and carbon compounds in the cometary dust (Joswiak et al. 2000). Theoretical work on light scattering by aggregate structures has shown that qualitative (and partially quantitative) fits to the observed scattering properties of cometary dust can be obtained using fractal aggregates of monomers composed of mixtures of silicates, organics, and (sometimes) metallic components. A short summary of these studies is presented in Mann et al. (2004). Following these results, we compositionally modeled the cometary dust using Mg-rich silicates and carbonaceous material as the main components.

The composition of the silicate component in cometary dust is usually investigated by studying the IR thermal emission spectra of comets. Because of the differences among comets, it is not possible to define a unique cometary composition, so we decided to use the composition of silicates derived from the analysis of comet Hale-Bopp IR spectra, which showed the strongest observed silicate emission features (Wooden et al. 1999), as representative of comets in general. Modeling the IR spectral dust features of Hale-Bopp with synthetic spectra based on laboratory studies, four components of dust silicates can be derived: amorphous and crystalline pyroxene, and amorphous and crystalline olivine (Wooden et al. 1999; Hayward et al. 2000;
Harker et al. 2002). The pyroxene formula is $\mathrm{Mg}_{x} \mathrm{Fe}_{1-x} \mathrm{SiO}_{3}$, where $x$ is the $\mathrm{Mg}$ number, $x=\frac{\mathrm{Mg}}{\mathrm{Mg}+\mathrm{Fe}}, x \in[0,1]$. Olivines are described by $\mathrm{Mg}_{2 y} \mathrm{Fe}_{2-2 y} \mathrm{SiO}_{4}$, with $y \in[0,1]$. Best fits to the observed IR spectra are found using the end-member enstatite $(x=1)$ as amorphous pyroxene (Wooden et al. 1999), pyroxene with $x \geq 0.9$ (Wooden et al. 1999) as representative of the crystalline phase, crystalline olivine with $y=0.9$ (Wooden et al. 1999) or $y \geq 0.95$ (Harker et al. 2002), and more Mgdeficient and Fe-rich $(y=0.5)$ amorphous olivine (Harker et al. 2002). Following these results, enstatite was chosen for both amorphous and crystalline pyroxene in the model (Davidsson $\&$ Skorov 2002). Amorphous olivine with $y=0.5$ and crystalline olivine with $y=0.95$ were taken into account for the olivine component. The refractive indices of the materials at the selected wavelengths were calculated by linearly interpolating the data obtained from laboratory studies. Glassy enstatite (Dorschner et al. 1995) was chosen as representative of both amorphous and crystalline enstatite (Davidsson \& Skorov 2002). The refractive indices of glassy $\mathrm{Mg}$-poor olivine with $y=0.5$ from Dorschner et al. (1995) were used for amorphous olivine. The crystalline olivine is a Mg-rich olivine with $y=0.95$ (Fabian et al. 2001). As far as the relative abundances of different silicate components are concerned, the works of Hayward et al. (2000) and Harker et al. (2002), corrected later in Harker et al. (2004), were taken into account. We assumed an average of the Hayward and Harker models as our cometary silicate composition. The resulting complex refractive indices of the composite material, calculated with the Maxwell-Garnett theory (Bohren \& Huffman 1987), are not very sensitive to this assumption. It leads to a silicate mixture composed of the following mass percentages: $30 \%$ amorphous pyroxene, $15 \%$ crystalline pyroxene, $40 \%$ crystalline olivine, and $15 \%$ amorphous olivine. The calculated refractive indices, $m$, and volume densities, $\rho$, for the silicate mixture are shown in Table $2 \mathrm{a}$. The optical and physical data of the single silicate components used are reported in Table 2, where AE is amorphous enstatite, $\mathrm{CE}$ is crystalline enstatite, $\mathrm{AO}$ is amorphous olivine, and $\mathrm{CO}$ is crystalline olivine.

The relative abundance of the various components containing carbon in cometary dust is still the subject of debate. The value used by different authors varies dramatically (see e.g. 
Table 2. Optical and physical properties of the materials used in the calculations at the wavelengths $0.535,0.6274$, and $1.5 \mu \mathrm{m}$.

\begin{tabular}{cccccccc}
\hline \hline & AE & CE & AO & CO & a & b & $\mathrm{c}$ \\
\hline $\mathrm{m}(0.5350)$ & $(1.58,2.2 \mathrm{E}-5)$ & $(1.58,2.2 \mathrm{E}-5)$ & $(1.76,0.089)$ & $(1.63,5.2 \mathrm{E}-6)$ & $(1.62,0.011)$ & $(1.88,0.423)$ & $(1.79,0.278)$ \\
$\mathrm{m}(0.6274)$ & $(1.57,2.5 \mathrm{E}-5)$ & $(1.57,2.5 \mathrm{E}-5)$ & $(1.75,0.069)$ & $(1.63,8.5 \mathrm{E}-6)$ & $(1.61,0.008)$ & $(1.92,0.378)$ & $(1.81,0.248)$ \\
$\mathrm{m}(1.5000)$ & $(1.54,4.6 \mathrm{E}-5)$ & $(1.54,4.6 \mathrm{E}-5)$ & $(1.77,0.060)$ & $(1.62,3.9 \mathrm{E}-5)$ & $(1.60,0.007)$ & $(2.01,0.164)$ & $(1.87,0.109)$ \\
$\rho\left(\mathrm{g} \mathrm{cm}^{-3}\right)$ & 2.71 & 2.71 & 3.71 & 3.27 & 3.04 & 1.60 & 2.10 \\
\hline
\end{tabular}

Table 3. Bulk density in $\mathrm{g} \mathrm{cm}^{-3}$ of the compositions considered in the model for different $N$ with PCA (left) and BPCA (right) dust particles.

\begin{tabular}{cccc|ccc}
\hline \hline$N$ & $\mathrm{a}$ & $\mathrm{b}$ & $\mathrm{c}$ & $\mathrm{a}$ & $\mathrm{b}$ & $\mathrm{c}$ \\
\hline 5 & 0.59 & 0.32 & 0.42 & 0.93 & 0.50 & 0.65 \\
15 & 0.15 & 0.08 & 0.10 & 0.55 & 0.30 & 0.38 \\
30 & 0.06 & 0.03 & 0.04 & 0.47 & 0.26 & 0.33 \\
50 & 0.03 & 0.02 & 0.02 & 0.45 & 0.24 & 0.31 \\
100 & 0.01 & 0.01 & 0.01 & 0.42 & 0.23 & 0.30 \\
\hline
\end{tabular}

Greenberg 1998; Fomenkova 1999; Kimura et al. 2003). We assumed the organic refractories (carbon mixed with other elements) in our calculations as representative of the carbonaceous species. Since it is still unclear if interstellar dust remains unmodified during its incorporation into comets, the assumption was made that the composition of organic refractories modeled in interstellar dust is also representative of cometary dust. It is believed that the organic refractory material originally started out as ices of simple chemical compounds that have undergone billions of years of UV photoprocessing in interstellar space, changing the ice mixture into a carbon-rich and oxygen-poor refractory material containing many different organic molecules (Greenberg \& Hage 1990). We used the optical constants calculated by Jenniskens (1993) with laboratory measurements of the organic residue resulting from heavily UV-irradiated ice mixtures. The values of $m$ and $\rho$ of the organic refractory residue were derived by linearly interpolating the published data and are shown in Table $2 b$.

From PUMA-1 data, the modeling of dust scattering, of nuclei composition, and of IR spectra, it has been found that the overall mass ratio of silicates to organics in comets is $M_{\text {sil }} / M_{\mathrm{or}} \sim$ 1-2 (Fomenkova \& Chang 1993; Kimura et al. 2003; Greenberg \& Hage 1990; Hanner \& Hayward 2003). We decided to investigate the effects of different composition on the scattered light using particles composed of pure silicate and organic compounds, plus particles composed of an intimate mixture of silicates and organics. The mass ratio of the mixed component was assumed to be $M_{\mathrm{sil}} / M_{\mathrm{or}}=1$. The refractive indices of the mixed component were calculated with the Maxwell-Garnett theory. In Table $2 c$ the optical and physical properties of cometary dust are shown for the chosen mixture of silicates and organics at the chosen wavelengths.

The calculated bulk density, $\rho_{\mathrm{B}}$, of the dust particles, related to $\rho$ and $p$ through $\rho_{\mathrm{B}}=\rho(1-p)$, are shown in Table 3. As in Table 2, Table 3 (a) indicates the silicate mixture, (b) the organics, and (c) the silicate-organic mixture. Most of the BPCA and small PCA particles used in the model are characterized by bulk porosity that falls in the range determined by Fulle et al. (2000) in a re-analysis of the Giotto data $\left([0.05-0.5] \mathrm{g} \mathrm{cm}^{-3}\right.$ with a preferred value of $0.1 \mathrm{~g} \mathrm{~cm}^{-3}$ ).

\section{The scattering analysis}

Several types of effects were investigated:

- the effects of different monomer radii, $R_{\mathrm{m}}$;

- the effects of different numbers of monomers, $N$;
- the effects of different compositions;

- the effects of different morphologies (i.e. different $D_{\mathrm{f}}$ ).

The effects on $P, \Phi_{S}$, and $A$ of single dust particles and averaged over a size distribution were studied. We neglected multiple scattering because the cometary coma is generally optically thin (Petrova et al. 2001).

The scattering calculations were performed using the DDA method and the code DDSCAT.5a10 by Draine \& Flatau (2000). The DDA method replaces the scattering target with an array of point dipoles. It then solves the electromagnetic scattering problem for an incident periodic wave interacting with this array of dipoles (Draine \& Flatau 2000). The results presented are averaged over many random orientations of the single dust particle with respect to the propagation direction of the incident wave, or, equivalently, over a collection of identical particles having different orientations in the 3D space. In the calculations, 6859 orientations, corresponding to 19 values of each of the three angles defining the particle orientation in the code, have been chosen for particles characterized by $N \leq 50$. The number of orientations for particles with $N=100$ was set to $9^{3}=729$, in order to save precious computational time. The calculations were performed fulfilling the DDSCAT condition to obtain accurate results: $|m| k d<0.5$, where $k$ is the wave number of the incident wavelength and $d$ the dipole size (Draine \& Flatau 2000). Each monomer is described by $\sim 900$ dipoles.

The polarization for single dust particles, considering unpolarized incident sunlight, is calculated with (Bohren \& Huffman 1987):

$P(\theta)=-\frac{S_{12}(\theta)}{S_{11}(\theta)}$

where $\theta$ is the scattering angle and $S_{11}, S_{12}$ are elements of the Mueller scattering matrix. The phase function $\Phi_{S}$ is:

$\Phi_{S}=\frac{1}{C_{\text {sca }}} \frac{S_{11}}{k^{2}}$

where $C_{\text {sca }}$ is the scattering cross section, and $\Phi_{S}$ is normalized on the solid angle $\Omega$ such that (Divine et al. 1986):

$\int_{4 \pi} \Phi_{S} \mathrm{~d} \Omega=2 \pi \int_{0}^{\pi} \Phi_{S}(\theta) \sin (\theta) \mathrm{d} \theta=1$

The geometric albedo, $A$, is calculated with (Divine et al. 1986):

$A=\pi Q_{\mathrm{sca}} \Phi_{S}(\pi)$

where $Q_{\text {sca }}$ is the scattering efficiency.

Once a size distribution is applied, the $S_{i, j}$ elements in Eqs. (1) and (2) have to be calculated with (Petrova et al. 2001):

$S_{i, j}(\theta)=\frac{\int_{r_{1}}^{r_{2}} C_{\mathrm{sca}}(r) S_{i j}(\theta, r) n(r) \mathrm{d} r}{\int_{r_{1}}^{r_{2}} C_{\mathrm{sca}}(r) n(r) \mathrm{d} r}$ 
where $S_{i j}(\theta, r)$ are the elements of the scattering matrix for a particle with radius $r$ and scattering cross section $C_{\text {sca }}(r)$. The chosen size distribution provides the number $n(r)$ of particles characterized by the radius $r . r_{1}$ and $r_{2}$ are the cuts of the size distribution. The variable $Q_{\text {sca }}$ in Eq. (4) has to be substituted with (Hansen \& Travis 1974):

$Q_{T \text { sca }}=\frac{\int_{r_{1}}^{r_{2}} \pi r^{2} Q_{\text {sca }}(r) n(r) \mathrm{d} r}{\int_{r_{1}}^{r_{2}} \pi r^{2} n(r) \mathrm{d} r}$

where $Q_{\text {sca }}(r)$ are the scattering efficiencies of the single particles.

Because we have irregular particles, the particle radius $r$ in Eqs. (5) and (6) has been substituted with the so-called effective radius $r_{\text {eff }}$, i.e. the radius of a sphere having the same volume as the irregular particle (Draine \& Flatau 2000). In that way, the cuts of the size distribution correspond to $r_{1} \sim 0.2 \mu \mathrm{m}$ and $r_{2} \sim$ $0.7 \mu \mathrm{m}$, depending on the $R_{\mathrm{m}}$ value.

The choice of the size of the dust particles used in the simulations is affected by the limits of the scattering program used and the computers available for the calculations. DDSCAT has been designed to provide precise results for particles that are comparable in size to the incident wavelength. Moreover, scattering simulations of the PCA-elongated particles require a larger amount of computer memory to run with respect to more compact BPCA particles with the same effective radius. It follows that PCA particles can be analyzed only for small $N$. The scattering simulations for BPCAs was therefore run for $N$ up to 100 (corresponding to $r_{\mathrm{eff}} \sim 0.7 \mu \mathrm{m}$ and particle size parameter, calculated using $r_{\mathrm{eff}}, X \sim 8$, depending on the value of $R_{\mathrm{m}}$ ), while it was possible to run PCA simulations only up to $N \sim 30$ $\left(r_{\text {eff }} \sim 0.5 \mu \mathrm{m}\right.$ and $\left.X \sim 5\right)$.

\subsection{The effects of different monomer radius $R_{m}$}

It has been already shown that the size of the constituent monomers plays an important role in determining the scattering properties of the entire dust particle, in both theoretical (e.g. Kimura 2001; Petrova et al. 2004; Lasue \& Levasseur-Regourd 2006) and experimental works (Hadamcik et al. 2006). To investigate the effects of $R_{\mathrm{m}}$, we ran simulations with the monomer radius in the range [0.13-0.16] $\mu \mathrm{m}$. The results obtained in this small size range provide the best qualitative fits to the observed dust scattering properties. Sample results are presented in Fig. 2 $(N=5)$ and Fig. $3(N=100)$ for silicate and organic BPCA particles. The mixed composition gives results closer to organics.

In Fig. 2, if $R_{\mathrm{m}}$ is small compared to the incident wavelength (i.e. if the monomer size parameter $X_{\mathrm{m}} \lesssim 1.5$ ), the polarization curves are characterized by a bell shape, a maximum at intermediate $\alpha$, and always positive values (Figs. 2a,c,e with $R_{\mathrm{m}}=0.13 \mu \mathrm{m}$ ), somehow resembling the Rayleigh scattering behavior. Increasing $R_{\mathrm{m}}$ (equivalently increasing $X_{\mathrm{m}}$ ) decreases $P_{\max }$, and it often moves towards larger $\alpha$; the negative branch, if present, becomes wider and the inversion angle moves towards higher $\alpha$. These effects are clearly visible at the green wavelength (Figs. 2a,e). These results agree with Kimura (2001), Petrova et al. (2004), Lasue \& Levasseur-Regourd (2006), and Hadamcik et al. (2006). The PCA particles with $N=5$ give us similar results. The polarization differences produced by $R_{\mathrm{m}}$ are reduced with increasing $N$ (compare Figs. 2a,c,e with Figs. 3a,c,e), suggesting a possible convergence of the polarization shape for larger sizes. Therefore, $P$ is less affected by $R_{\mathrm{m}}$ changes with increasing $N$.
The polarization curves shown, especially Figs. 3a,c for silicates, suggest a possible interpretation of the observed three polarization classes of comets. If only a qualitative match with observations is taken into account, then the presence of a small negative branch and different values in the polarization maximum can be associated with different values of $R_{\mathrm{m}}$ for large particles.

The effect of $R_{\mathrm{m}}$ on the polarization provides an important diagnostic tool for determining the monomer size in real cometary dust. If the particles produce a small negative branch (as observed in cometary dust) in the visible, we expect to see a polarization curve with a wide negative branch, an inversion angle at high $\alpha$, and low $P_{\max }$ in the UV region, because of the $X_{\mathrm{m}}$ dependence on $\lambda$. The same particle should produce a Rayleigh-like curve in the IR. In order to confirm this hypothesis, we ran some test simulations in the IR at $1.5 \mu \mathrm{m}$. All these simulations show no evidence of any negative branch, the polarization curves resembling those of Rayleigh scatterers. This theoretical behavior seems to be confirmed by the IR observations of the Hale-Bopp polarization, which showed no negative branch (Jones \& Gehrz 2000). On the other hand, comet Halley did exhibit a negative branch in the IR (Brooke et al. 1987). The presence of a negative IR branch in comet Halley cannot be explained with our model.

The analysis of $R_{\mathrm{m}}$ effects shows that $\Phi_{S}$ in the forward direction (which corresponds to the maximum $\Phi_{S \max }$ ) increases with $R_{\mathrm{m}}$ for all particles sizes and every considered composition and wavelength (Figs. 2-3(b,d,f)). This agrees with results obtained when analyzing the behavior of the $S_{11}$ element, proportional to the scattered intensity, in the forward direction, independently of the considered shape or composition (Kimura 2001; Petrova et al. 2004). At intermediate and low $\alpha$, a unique trend is not visible. The differences in $\Phi_{S \max }$ due to different $R_{\mathrm{m}}$ increase with $N$ (i.e. compare Figs. 2b,d,f with Figs. 3b,d,f), suggesting no convergent behavior of the $\Phi_{S \max }$ at larger $N$. Taking into account that $R_{\mathrm{m}}=0.13$ and $0.15 \mu \mathrm{m}$, silicates show an increase in FSE with decreasing $R_{\mathrm{m}}$ at green wavelength and an increase in $B S E$ with $R_{\mathrm{m}}$ at both wavelengths. In all other cases, a clear trend is not visible. The differences in FSE and BSE because of $R_{\mathrm{m}}$ can rise to $\sim 30 \%$.

The geometric albedo values obtained from the simulations are plotted in Fig. 4, which shows the albedo obtained with $R_{\mathrm{m}}=0.13,0.15 \mu \mathrm{m}$ at green (a) and red (b) wavelengths. In Fig. 4, $A$ at the two wavelengths is compared for $R_{\mathrm{m}}=0.13$ (c) and $0.15 \mu \mathrm{m}(\mathrm{d})$. At the red wavelength, $A$ decreases when $R_{\mathrm{m}}$ increases for every composition (Fig. 4b). At the green wavelength, $A$ increases with $R_{\mathrm{m}}$ for silicates, has the opposite behavior for organics, and a clear trend is not visible for the mixed composition (Fig. 4a). The differences between albedos obtained with the two $R_{\mathrm{m}}$ values are higher in the red (compare Fig. $4 \mathrm{a}$ with Fig. 4b), and $A$ increases with $\lambda$ for every composition and $R_{\mathrm{m}}=0.13 \mu \mathrm{m}$ (Fig. $4 \mathrm{c}$ ). This is also true for organics and $R_{\mathrm{m}}=0.15 \mu \mathrm{m}$, while there is no clear trend for silicate and mixed compositions (Fig. 4d). The differences in the albedos at the two considered wavelengths decrease when $R_{\mathrm{m}}$ increases (see comparison between Figs. 4c and 4d).

Since $R_{\mathrm{m}}$ affects the scattering results both for small and large particles, it is obvious to conclude that the monomer radius size maintains its influence even when a size distribution is applied. We have confirmed that $R_{\mathrm{m}}$ plays an important role in determining the scattering properties of the fractal aggregates constituted by cluster of spheres. This role has to be taken into account when general conclusions about the scattering properties are made. 

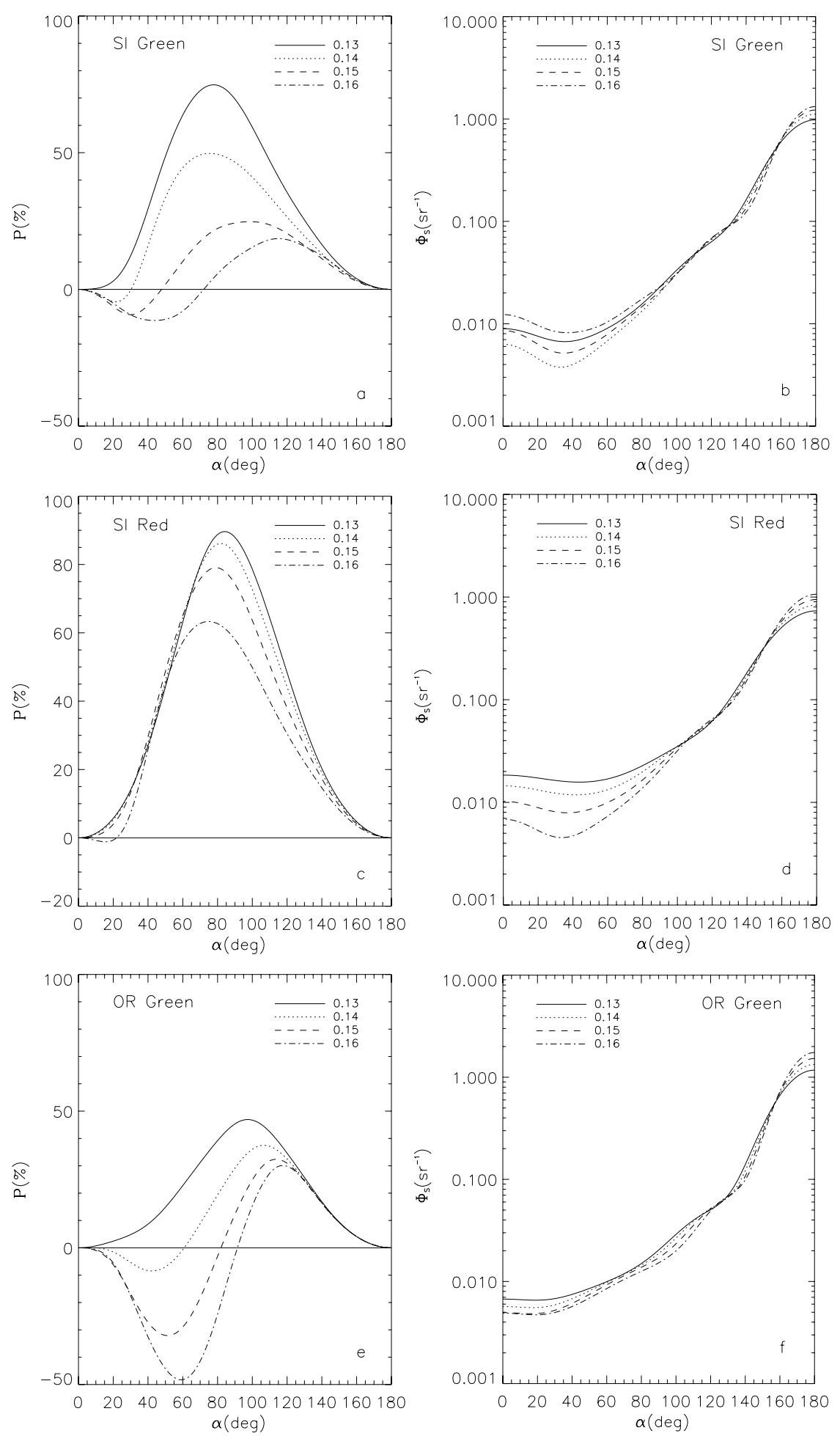

Fig. 2. Effect of $R_{\mathrm{m}}$ on the polarization (a), c), e)) and phase function (b), d), f)). $R_{\mathrm{m}}$ is in the range [0.13-0.16 $\mu$ m]. Lines show results with $R_{\mathrm{m}}=0.13$ (solid), 0.14 (dotted), 0.15 (dashed), $0.16 \mu \mathrm{m}$ (dash-dotted) for silicate (a)-d)) and organic (e), f)) compositions and $N=5$ at green $(\mathbf{a})$, b), e), f)) and red (c), d)) wavelengths.

\subsection{The effects of different numbers of monomers $N$}

The effects of different numbers of monomers (i.e. of the different size) have been studied for BPCA particles with $N=5,15$, 30, 50, 100. Samples of the results are shown in Figs. 5 and 6.

The polarization curves become less smooth with increasing $N$ (Figs. 5-6(a,c,e)). Results with higher $N(N \geq 30$, corresponding to $X \geq 5$ ) give better qualitative fits to the shape of the observed polarization negative branch. In the considered size range, the variations in the polarization produced by using different $R_{\mathrm{m}}$ are generally larger than the differences induced by changing $N$ (compare silicate curves in Figs. 5a and 5c). Once a negative branch is present, the different values of $N$ producing the negative branch do not strongly influence the polarization maximum, suggesting that the observed three polarization classes may not be explained in terms of a different particle size. 

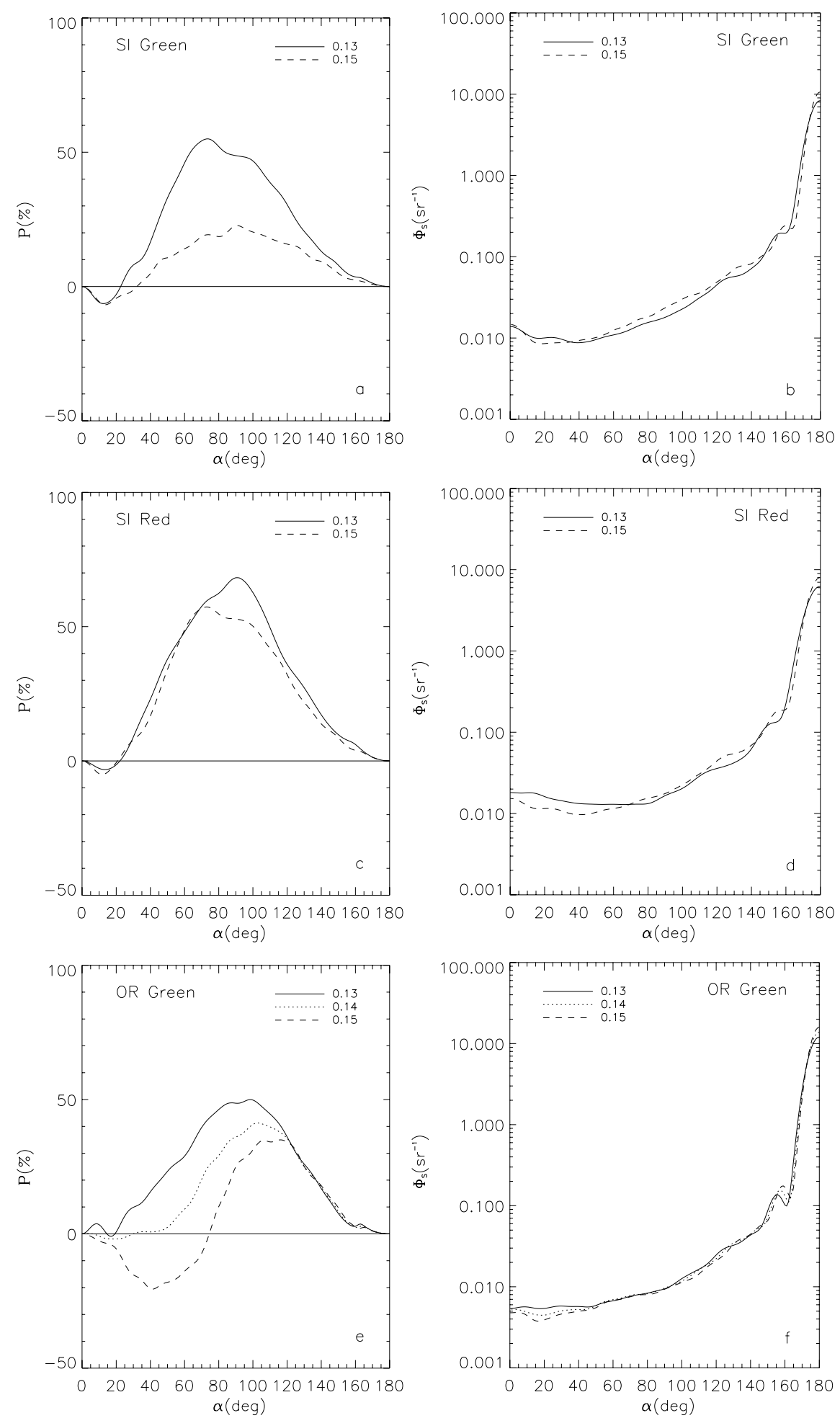

Fig. 3. Effect of $R_{\mathrm{m}}$ for particles with $N=100$. Lines show results with $R_{\mathrm{m}}=0.13$ (solid), 0.14 (dotted), $0.15 \mu \mathrm{m}$ (dashed) for silicates at green (a), b)) and red (c), d)) wavelengths and for organics at green $(\mathbf{e}), \mathbf{f})$ ).

These results agree with the conclusion that the overall trend of the polarization depends weakly on $N$, when the size of the particle is comparable to or larger than the incident wavelength (Kimura et al. 2003). We do not see the effects in $P$ with increasing $N$ shown in Kimura (2001), Mann et al. (2004), Petrova et al. (2004), and Vilaplana et al. (2006). Moreover, Petrova et al. (2004) also find that $P$ is less sensitive to $N$ when the size of the cluster is $\geqslant \lambda$, at least in the backscattering region.
Increasing $N$ leads to a corresponding increase in $\Phi_{S}$ in the forward direction for every considered composition and $R_{\mathrm{m}}$ (e.g. Figs. 5-6(b,d,f)). This trend is also visible for $S_{11}$ in Kimura et al. (2003), Mann et al. (2004), Petrova et al. (2004), and Vilaplana et al. (2006). Small aggregates $(N=5)$ always show a FSE double the one derived from cometary dust. The FSE values close to 10 are found for every composition with particles mainly in the range $N=30-100$ (corresponding to $X=4-7$ ). 

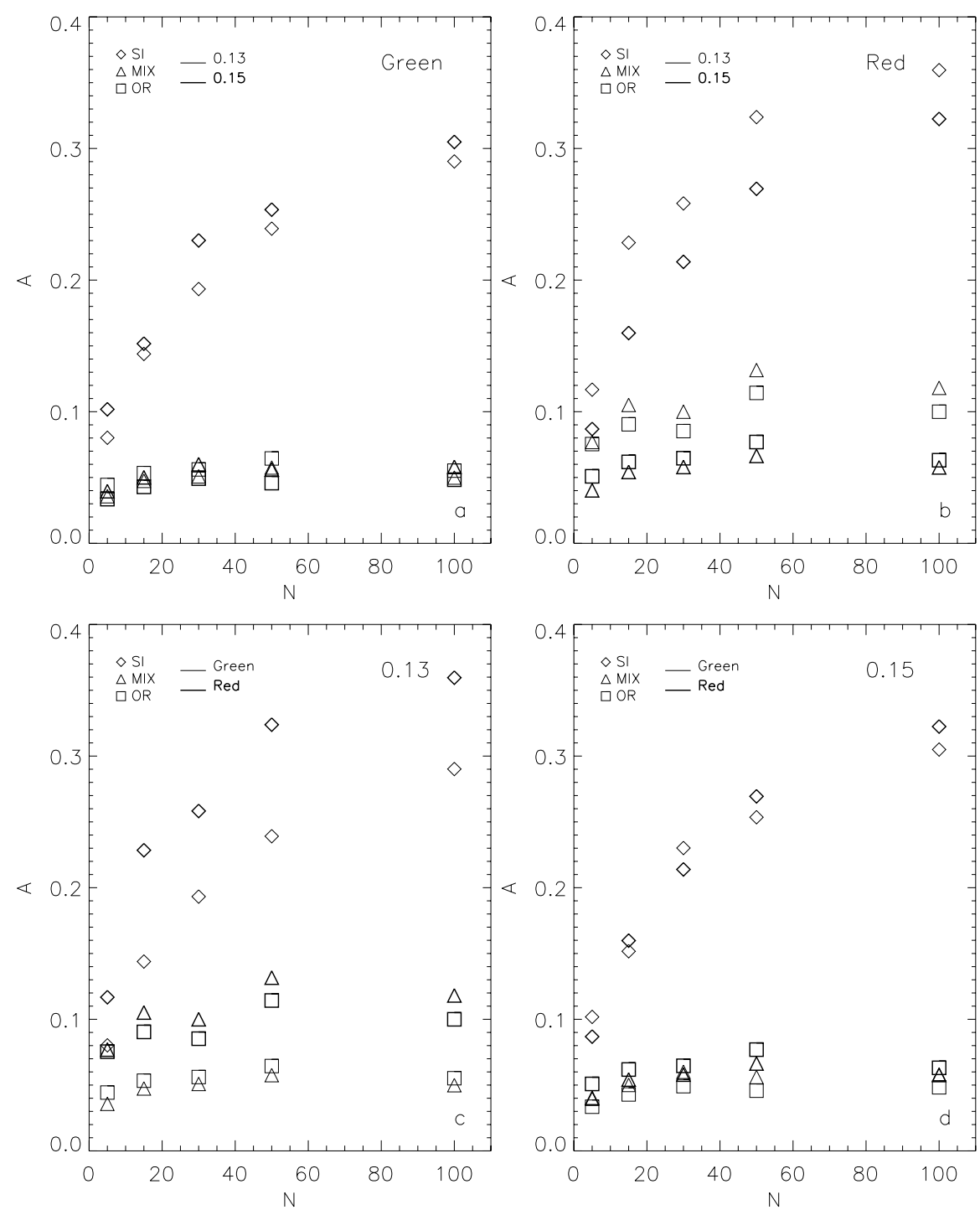

Fig. 4. Geometric albedo, $A$, of the used BPCA dust particles versus the number of constituent monomers, $N$. The results from the two different $R_{\mathrm{m}}$ are shown at green a) and red b) wavelengths. The albedo values for the two wavelengths are compared in $\mathbf{c}$ ) for $R_{\mathrm{m}}=0.13 \mu \mathrm{m}$ and in $\left.\mathbf{d}\right)$ for $R_{\mathrm{m}}=0.15 \mu \mathrm{m}$.

The influence of $N$ is also clearly visible for the $B S E$. Best fits to the observed value $B S E \sim 2$ are found for every composition with $N=30-50$ (corresponding to $X=5-6$ ).

From Fig. 4 it follows that the albedo is increasing with $N$ for small particles but then decreasing at larger sizes for organic and mixed compositions. The decrease is not visible in the silicates, but nevertheless, even in this case, the rate of increase decreases at high $N$.

These considerations about the polarization and phase function suggest that, among the size ranges we considered, the particles with size comparable to the incident wavelength $(X=5-7)$ provide the best qualitative fits to the observed scattering properties of cometary dust.

\subsection{The effects of different composition}

To have a more realistic view of the cometary dust, a size distribution has to be applied. We used a power-law size distribution, $n(r) \sim r^{-a}$, with exponent $a=2.6$, obtained from a re-analysis of the Giotto data by Fulle et al. (2000). The results are not sensitive to the choice of $a$ in the range $a=[2-3]$. The obtained polarization and phase function curves are plotted in Fig. 7 at the green and red wavelengths.

In the green, the polarization of the pure silicate composition qualitatively appears a better fit to the shape of the observed polarization curves for both $R_{\mathrm{m}}$ values $(0.13,0.15 \mu \mathrm{m})$, while the organic and mixed compositions do not show a qualitative fit to the negative branch in the entire investigated $R_{\mathrm{m}}$ range $(0.13-0.16 \mu \mathrm{m})$. In the red, the polarization is less affected by compositional effects. Nevertheless, the silicate class still provides the best qualitative fits. Since the organic and mixed compositions do not provide nice qualitative fits to the observed negative branch, we cannot say that different compositions may explain the different polarization classes. All the considered materials have $F S E \sim 10$. Silicates are also characterized by a backscattering enhancement close to 2 , while organics and mix composition have a lower $B S E=[1.0-1.3]$.

The carbonaceous and the mixed compositions have lower $\Phi_{S}$ in the backscattering direction than pure silicates and are characterized by lower albedo. Silicates give high geometric albedos $(A=0.20-0.22$ in the green and $A=0.22-0.26$ in the red). Organic and mixed compositions instead provide low 

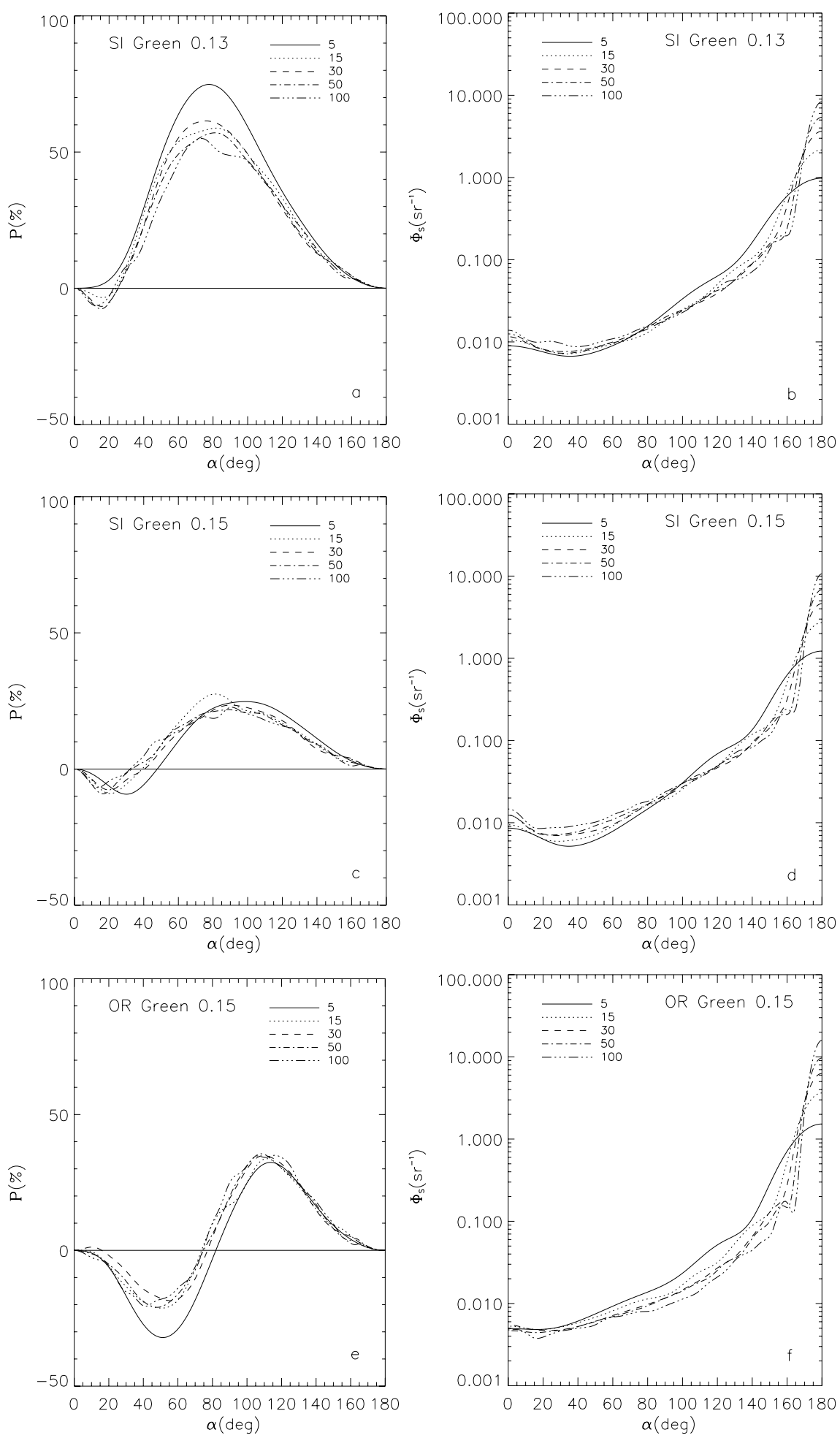

Fig. 5. Effects of $N$ on polarization (a), c), e)) and phase function (b), d), f)) curves for different compositions at green wavelength. Lines show results with $N=5$ (solid), 15 (dotted), 30 (dashed), 50 (dash-dotted), 100 (dash-dot-dotted) for silicate (a)-d)) and organic e), f)) compositions.

albedo values $(A=0.04-0.05$ in the green and $A=0.06-0.11$ in the red). It is interesting to note that in some cases, depending on $R_{\mathrm{m}}$, the mixed composition gives lower albedo than organics. The albedo for all the considered compositions and $R_{\mathrm{m}}$ increases with wavelength (i.e. it has a red color), once the results are averaged over the used size distribution. The albedo red color agrees with observational IR cometary data summarized in Hanner \& Newburn (1989). We found that the albedo differences at the two wavelengths decrease with increasing $R_{\mathrm{m}}$.
From the analysis of the results, we found that there is not a unique value of $R_{\mathrm{m}}$ that produces, for a fixed composition, identical shapes of the polarization negative branch at green and red wavelengths as is instead observed in cometary dust (LevasseurRegourd \& Hadamcik 2001). Nevertheless, if we combine results from different $R_{\mathrm{m}}$ in the two wavelengths, corresponding to similar $X$ values of the particles with the same $N$ constituting the size distribution, some useful information can be derived. We present the case of silicates in the green with $R_{\mathrm{m}}=0.13 \mu \mathrm{m}$ and 

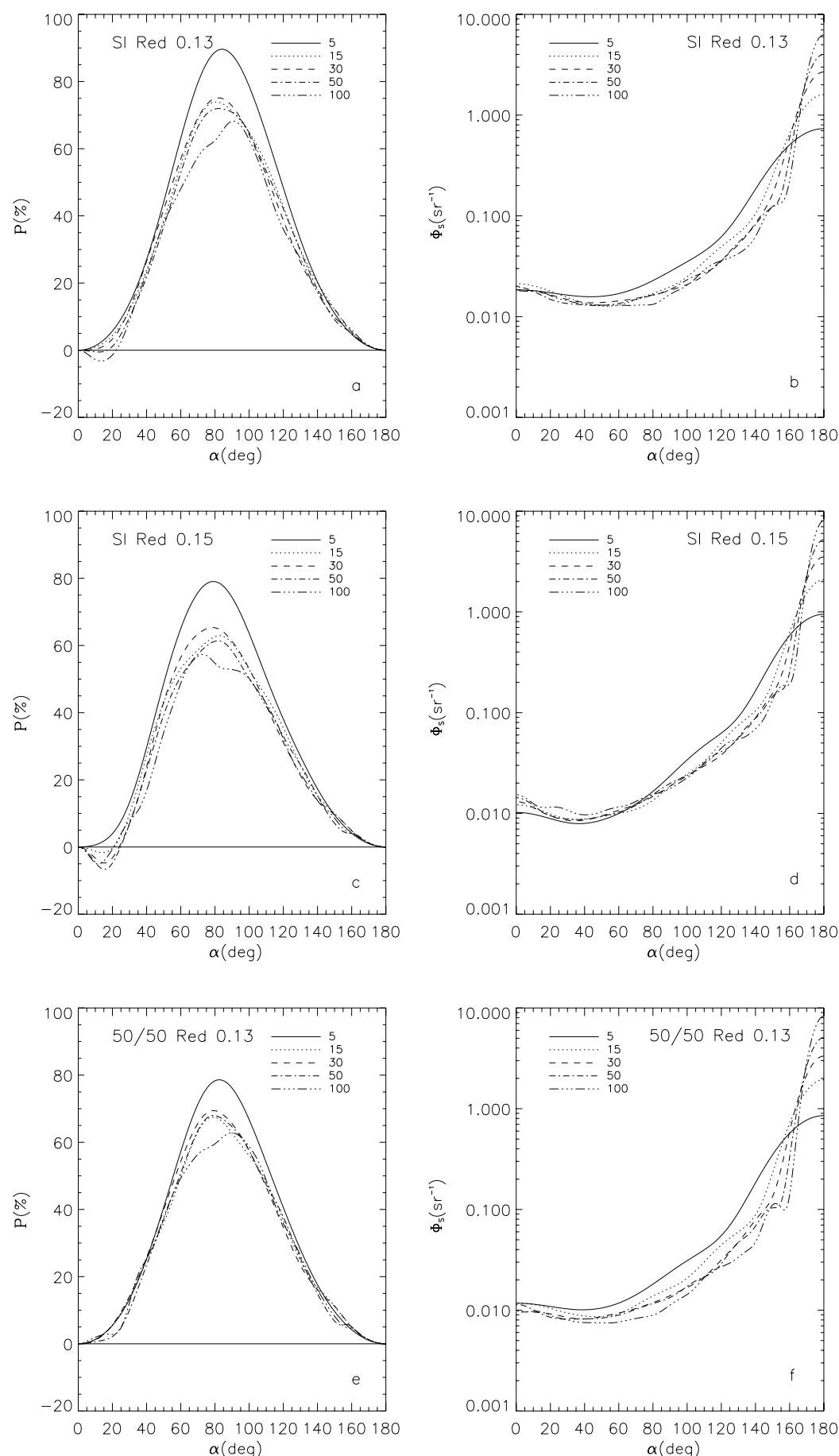

Fig. 6. Effects of $N$ at the red wavelength. Lines show results with $N=5$ (solid), 15 (dotted), 30 (dashed), 50 (dash-dotted), 100 (dash-dot-dotted) for silicate (a)-d)) and mixed (e), f)) compositions.

in the red with $R_{\mathrm{m}}=0.15 \mu \mathrm{m}$. In Fig. 8, the comparison between polarization and phase function values in the two $\lambda$ is shown. If the observed polarization in the red and green band is compared (e.g. Levasseur-Regourd et al. 2004), it can be derived that for $\alpha>30^{\circ}-40^{\circ}$, the polarization has a red color that increases up to intermediate phase angles and decreases for larger $\alpha$. The percentage polarization color, $P_{\text {red }}-P_{\text {green }}$, is plotted in Fig. 8c. In our case the color is not flat below $\alpha \sim 40^{\circ}$, as it should be. We find the percentage red color increasing up to $1.5 \%$ around the inversion angle, then decreasing again until $\alpha \sim 40^{\circ}$. At larger phase angles, the behavior of the polarization red color matches the observed one, increasing until intermediate $\alpha$ and decreasing toward larger phase angles. The phase function color, $\Phi_{S \text { red }}-\Phi_{S \text { green }}$, behavior is more complex (Fig. 8d). Color differences are only discernable at high phase angles. The color is slightly red from low to intermediate phase angles and then slightly green up to $\alpha \sim 160^{\circ}$. At larger phase angles, the color becomes red in the range $\alpha \sim 160^{\circ}-170^{\circ}$, becoming monotonically green after that.

In conclusion, we have not found a composition that qualitatively fits all the observed scattering properties of cometary dust for this model and its set of parameters. Silicates provide 

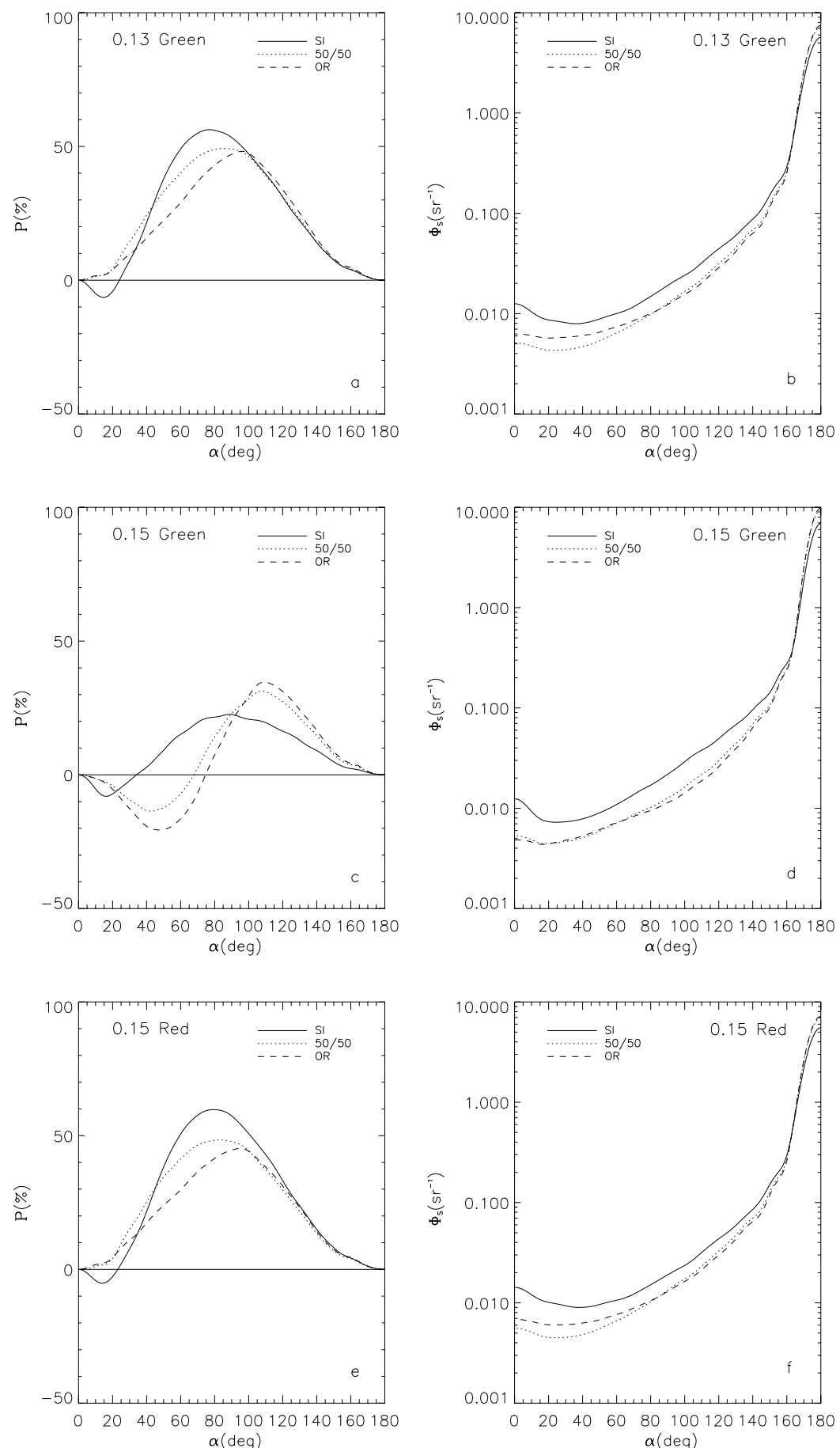

Fig. 7. Effects of composition at green (a)-d)) and red (e), f)) wavelength, considering a size distribution. Lines show results with silicate (solid), mixed (dotted), and organic (dashed) compositions for $\left.\left.R_{\mathrm{m}}=0.13 \mu \mathrm{m}(\mathbf{a}), \mathbf{b}\right)\right)$ and $\left.R_{\mathrm{m}}=0.15 \mu \mathrm{m}(\mathbf{c})-\mathbf{f}\right)$ ).

interesting fits to the polarization and phase function curves, also reproducing the polarization red color over a large angular range, but they are characterized by albedo values that are too high. Organics and 1:1 mixture compositions have a low albedo value but do not provide good qualitative fits of the polarization and phase function.

\subsection{The effects of different morphological classes}

The effects of the different morphological classes (i.e. of fractal mass dimension $D_{\mathrm{f}}$ ) have been investigated by studying the scattering properties of elongated PCA and more compact BPCA particles. Because of the limitations of available computers, the comparative analysis was carried out only up to $N=30$ $\left(r_{\text {eff }} \sim 0.5 \mu \mathrm{m}\right)$. Sample results are presented in Fig. 9 and are averaged over the used size distribution. The BPCA particles only up to $N=30$ were considered to provide a comparison with PCAs over the same size range.

In order to estimate the differences in the scattering behavior of BPCAs and PCAs, we calculated the integral over the entire angular range $\left(\alpha=0^{\circ}-180^{\circ}\right)$ of the absolute value of the difference between two scattering curves $\left(P\right.$ or $\left.\Phi_{S}\right)$ having the same 

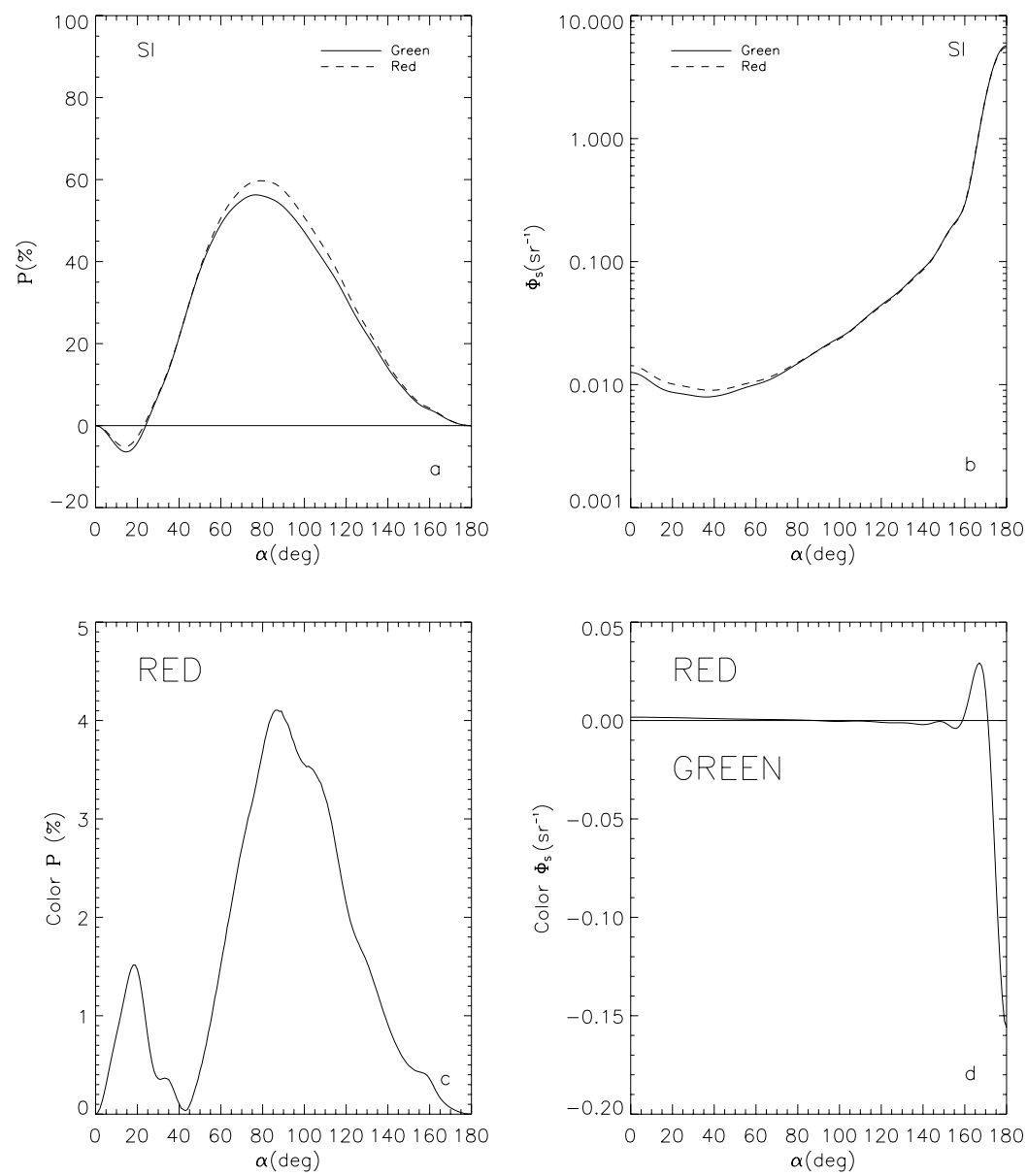

Fig. 8. Color effects on polarization and phase function for a silicate composition. a) shows the polarization values at the two wavelengths (the solid line stands for green and $R_{\mathrm{m}}=0.13 \mu \mathrm{m}$ and the dashed line for red and $R_{\mathrm{m}}=0.15 \mu \mathrm{m}$ ), b) the corresponding phase functions. The polarization and phase function colors are plotted in $\mathbf{c}$ ) and $\mathbf{d}$ ), respectively. The results are averaged over the used size distribution.

composition, $N$ and $R_{\mathrm{m}}$, but corresponding to different morphological classes. This parameter shows clearly that the difference in $\Phi_{S}$ increases with $N$. The behavior of the polarization is more complex. For organics, the difference in $P$ increases with $N$, while for silicate and mixed compositions this is happening only in the $50 \%$ of the cases. In the other cases the maximum difference is reached with $N=15$, and then it slowly decreases for $N=30$.

We could not define which morphological class fits the observed polarization properties best. The polarization maximum differences due to the morphological class are small (especially in the green, Figs. 9a,c), once $R_{\mathrm{m}}$ is fixed. This suggests that differences in the morphological class do not explain the different polarization classes. Our last conclusion agrees with the majority of previous works, which state that the morphology of the dust particles does not play an important role in determining the shape of the polarization (Kimura 2001; Kimura et al. 2003; Kolokolova et al. 2006; Mann et al. 2004; Lasue \& LevasseurRegourd 2006; Vilaplana et al. 2006). Petrova et al. (2004) instead find some trends in the morphological influence on $P$.

A clearer trend can be found in the phase function and albedo (Figs. 9b,d,f). The PCA particles have higher $\Phi_{S \max }$ than BPCAs in the forward direction for every composition and $R_{\mathrm{m}}$. This time, our conclusion agrees with Petrova et al. (2004), where the $S_{11}$ element for sparse clusters is described as larger in almost the whole angular range with respect to compact aggregates. On the other hand, Kimura (2001), Kimura et al. (2003), Mann et al. (2004), Moreno et al. (2006), and Vilaplana et al. (2006) instead find that the morphology does not strongly influence the shape of the scattered intensity for irregular particles. The PCAs have lower $\Phi_{S}$ in the backscattering region for the silicate composition (in every $\lambda$ and $R_{\mathrm{m}}$ ), providing a lower value of the albedo and somehow resembling the behavior of more compact darker grains. Organic and mixed compositions do not provide a clear trend. The PCA particles always show $F S E_{\mathrm{PCA}} \sim 20$, larger than more compact aggregates $\left(F S E_{\mathrm{BPCA}}<15\right)$. The two different classes have comparable values of $B S E$.

From the comparison of the scattering properties of elongated and compact aggregates, we conclude that compact particles fit the observed scattering properties slightly better. Since PCAs resemble pre-planetary dust particles (probably similar to the ones that formed comets) and more compact BPCAs seem to fit the observed properties better, this may indicate a morphological metamorphism of cometary dust from the time of formation to the release from the nucleus and the subsequent processing in the solar environment. To derive definitive conclusions, the investigation at larger sizes is required, where the differences between the two morphological classes arise for the phase function and, in some cases, for the polarization. 

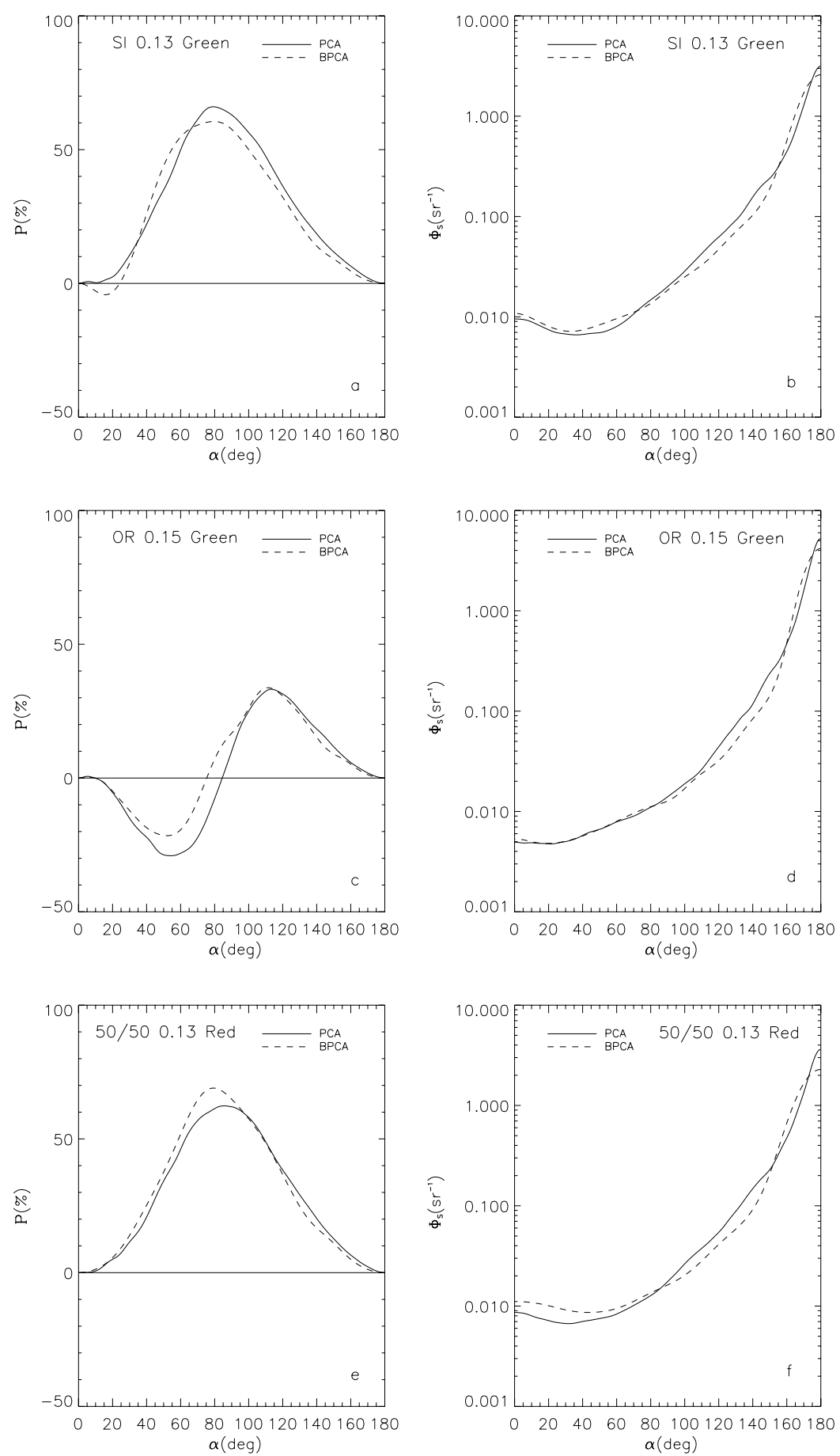

Fig. 9. Effects of $D_{\mathrm{f}}$ on the polarization $\left.\left.(\mathbf{a}), \mathbf{c}\right), \mathbf{e}\right)$ ) and phase function $\left.\left.(\mathbf{b}), \mathbf{d}\right), \mathbf{f}\right)$ ) curves at green (a)-d)) and red (e), f)) wavelengths. Elongated PCA (solid) and compact BPCA (dashed) particles up to $N=30$ are considered. Results are plotted for silicate with $R_{\mathrm{m}}=0.13 \mu \mathrm{m}(\mathbf{a})$, b)), organic with $\left.R_{\mathrm{m}}=0.15 \mu \mathrm{m}(\mathbf{c}), \mathbf{d}\right)$ ), and mixed compositions with $\left.R_{\mathrm{m}}=0.13 \mu \mathrm{m}(\mathbf{e}), \mathbf{f}\right)$ ). The results are averaged over the used size distribution.

\section{Summary and conclusions}

The scattering behavior of irregular, highly porous dust particles in cometary coma by means of the fractal aggregates of spherical grains have been modeled with the DDA theory. The scattering properties of extremely elongated dust particles (probably similar to the ones that formed comets) were investigated and compared with more compact aggregates. Particles up to $r_{\text {eff }} \sim 0.7 \mu \mathrm{m}$ and $X \sim 8$ (compact) and to $r_{\text {eff }} \sim 0.5 \mu \mathrm{m}$ and $X \sim 5$ (elongated) were used. Several compositional and morphological effects were investigated. Our model shows results that in many aspects agree with previous theoretical and experimental works. A complete match is impossible to obtain because in theoretical modeling only a few restricted areas of the model parameters can be investigated, and the trends found by different authors sometimes disagree. In summary, we find that:

1. The results are strongly dependent on the choice of the monomer radius $R_{\mathrm{m}}$. Values around $0.13-0.16 \mu \mathrm{m}$ are optimum in the considered size range. 
2. The particles comparable in size to the incident wavelength $(X=5-7)$ give better qualitative fits to the observed scattering properties.

3. There is no composition that qualitatively fits all the observed properties. Silicates provide good fits in the polarization and in the phase function, also to a large extent reproducing the red polarization color, but they are characterized by a high albedo. Organics and 1:1 mixture give low albedos, but their phase functions have a small backscattering enhancement. Moreover, they do not provide a good qualitative fit to the observed polarization, at least for the considered $R_{\mathrm{m}}$ range.

4. Compact particles provide slightly better qualitative fits than elongated aggregates. This may prove a morphological metamorphism since the time of formation, because, as shown in the CODAG experiment, primitive pre-planetary particles forming comets were probably extremely elongated. Further investigations at larger sizes, where differences between the two classes do in some cases arise, are needed.

5. The differences in $R_{\mathrm{m}}$ may provide an explanation for the different observed polarization classes, if the particles are modeled with clusters of spheres. The classes cannot be explained in terms of different size, morphological class, and maybe composition.

We plan to perform further investigations at larger sizes, in order to constrain the nature of cometary dust much more. Moreover, interesting compositional and morphological hints for future theoretical works will probably be provided by the analysis of the cometary sample collected by the Stardust mission, arrived in January 2006.

Acknowledgements. Authors would like to thank: J. Blum for his information about dust aggregation experiments; T. Kozasa for having provided the original BPCA code; B. Davidsson for his suggestions about dust composition; B. T. Draine and P. J. Flatau for their public scattering code DDSCAT and for suggestions about its use; S. Casotto, W. Benz, T-M. Ho, S. Fornasier, and the Bern Planetary Imaging Group for having provided precious computational time through the use of their computer resources. We are grateful to the anonymous referee for the useful suggestions that helped improve the quality of this paper. I. Bertini is indebted to the International Space Science Institute (ISSI) of Bern and to L.-M. Lara of the Istituto de Astrofisica de Andalucia of Granada for their financial support during visiting scientist periods.

\section{References}

Bohren, C. F., \& Huffman, D. R. 1987, Absorption and Scattering of Light by Small Particles, Wiley Science Paperback Series

Brooke, T. Y., Knacke, R. F., \& Joyce, R. R. 1987, A\&A, 187, 621

Brownlee, D. E., Joswiak, D. J., Love, S. G., et al. 1994, in Lunar and Planetary Institute Conference Abstracts, 185

Chernova, G. P., Kiselev, N. N., \& Jockers, K. 1993, Icarus, 103, 144

Colangeli, L., Mennella, V., di Marino, C., Rotundi, A., \& Bussoletti, E. 1995, A\&A, 293, 927

Colangeli, L., Mennella, V., Rotundi, A., Palumbo, P., \& Bussoletti, E. 1996, A\&A, 312, 643

Davidsson, B. J. R., \& Skorov, Y. V. 2002, Icarus, 156, 223

Divine, N., Fechtig, H., Gombosi, T. I., et al. 1986, Space Sci. Rev., 43, 1

Dorschner, J., Begemann, B., Henning, T., Jaeger, C., \& Mutschke, H. 1995, A\&A, 300, 503
Draine, B. T., \& Flatau, P. J. 2000, User Guide for the Discrete Dipole Approximation Code DDSCAT (Version 5a10)

Fomenkova, M. N. 1999, Space Sci. Rev., 90, 109

Fomenkova, M., \& Chang, S. 1993, in Lunar and Planetary Institute Conference Abstracts, 501

Fulle, M., Levasseur-Regourd, A. C., McBride, N., \& Hadamcik, E. 2000, AJ, 119,1968

Gehrz, R. D., \& Ney, E. P. 1992, Icarus, 100, 162

Greenberg, J. M. 1998, A\&A, 330, 375

Greenberg, J. M., \& Hage, J. I. 1990, ApJ, 361, 260

Hadamcik, E., Renard, J.-B., Levasseur-Regourd, A. C., \& Lasue, J. 2006, J. Quant. Spec. Radiat. Transf., 100, 143

Hansen, J. E. \& Travis, L. D. 1974, Space Sci. Rev., 16, 527

Hanner, M. S., \& Newburn, R. L. 1989, AJ, 97, 254

Hanner, M. S., \& Hayward, T. L. 2003, Icarus, 161, 164

Hanner, M. S., Tedesco, E., Tokunaga, A. T., et al. 1985, Icarus, 64, 11

Harker, D. E., Wooden, D. H., Woodward, C. E., \& Lisse, C. M. 2002, ApJ, 580, 579

Harker, D. E., Wooden, D. H., Woodward, C. E., \& Lisse, C. M. 2004, ApJ, 615, 1081

Hayward, T. L., Hanner, M. S., \& Sekanina, Z. 2000, ApJ, 538, 428

Jenniskens, P. 1993, A\&A, 274, 653

Jessberger, E. K. 1999, Space Sc. Rev., 90, 91

Jessberger, E. K., Kissel, J., Fechtig, H., \& Krueger, F. R. 1986, in ESA SP-249: Comet Nucleus Sample Return Mission, ed. O. Melita, 27

Jones, T. J., \& Gehrz, R. D. 2000, Icarus, 143, 338

Joswiak, D. J., Brownlee, D. E., Pepin, R. O., \& Schlutter, D. J. 2000, in Lunar and Planetary Institute Conference Abstracts, 1500

Keller, H. U., Barbieri, C., Lamy, P., et al. 2006, Space Sci. Rev., in press

Kelley, M. S., Woodward, C. E., Jones, T. J., Reach, W. T., \& Johnson, J. 2004, AJ, 127, 2398

Kimura, H. 2001, J. Quant. Spec. Radiat. Transf., 70, 581

Kimura, H., Kolokolova, L., \& Mann, I. 2003, A\&A, 407, L5

Kiselev, N. N., Jockers, K., \& Bonev, T. 2004, Icarus, 168, 385

Kissel, J., \& Krueger, F. R. 1987, Nature, 326, 755

Kissel, J., Krueger, F. R., Silén, J., \& Clark, B. C. 2004, Science, 304, 1774

Kolokolova, L., Hanner, M. S., Levasseur-Regourd, A.-C., \& Gustafson, B. Å. S. 2004, Comets II, 577

Kolokolova, L., Kimura, H., Ziegler, K., \& Mann, I. 2006, J. Quant. Spec. Radiat. Transf., 100, 199

Kozasa, T., Blum, J., \& Mukai, T. 1992, A\&A, 263, 423

Kozasa, T., Blum, J., Okamoto, H., \& Mukai, T. 1993, A\&A, 276, 278

Krause, M., \& Blum, J. 2004, Phys. Rev. Lett., 93, 021103

Lasue, J., \& Levasseur-Regourd, A. C. 2006, J. Quant. Spec. Radiat. Transf., 100,220

Lawler, M. E., \& Brownlee, D. E. 1992, Nature, 359, 810

Levasseur-Regourd, A. C., \& Hadamcik, E. 2001, in Meteoroids 2001 Conference, ed. B. Warmbein, ESA SP-495 587

Levasseur-Regourd, A. C., Hadamcik, E., \& Renard, J. B. 1996, A\&A, 313, 327 Levasseur-Regourd, A. C., Hadamcik, E., Lasue, J., Renard, J. B., \& Worms, J. C. 2004, in The New Rosetta Targets. Observations, Simulations and Instrument Performances, ed. L. Colangeli, E. Mazzotta Epifani, \& P. Palumbo, ASSL, 311, 111

Mann, I., Kimura, I., \& Kolokolova, L. 2004, J. Quant. Spec. Radiat. Transf., 89, 291

Millis, R. L., Ahearn, M. F., \& Thompson, D. T. 1982, AJ, 87, 1310

Moreno, F., Muñoz, O., Vilaplana, R., \& Molina, A. 2003, ApJ, 595, 522

Moreno, F., Vilaplana, R., Muñoz, O., Molina, A., \& Guirado, D. 2006, J. Quant. Spec. Radiat. Transf., 100, 277

Ney, E. P. 1982, in Comet Discoveries, Statistics, and Observational Selection, ed. L. L. Wilkening, IAU Colloq., 61, 323

Ney, E. P., \& Merrill, K. M. 1976, Science, 194, 1051

Petrova, E. V., Jockers, K., \& Kiselev, N. N. 2001, Solar System Res., 35, 57

Petrova, E. V., Tishkovets, V. P., \& Jockers, K. 2004, Solar System Res., 38, 309

Tokunaga, A. T., Golisch, W. F., Griep, D. M., Kaminski, C. D., \& Hanner, M. S. 1986, AJ, 92, 1183

Vilaplana, R., Moreno, F., \& Molina, A. 2006, J. Quant. Spec. Radiat. Transf., 100,415

Wooden, D. H., Harker, D. E., Woodward, C. E., et al. 1999, ApJ, 517, 1034

Wurm, G., \& Blum, J. 1998, Icarus, 132, 125 